Bull. Mater. Sci., Vol. 36, No. 4, August 2013, pp. 517-520. (C) Indian Academy of Sciences.

\title{
Dependence of thermal conductivity in micro to nano silica
}

\author{
VANGALA DHANUNJANA CHARI ${ }^{\mathrm{a}, \mathrm{b}, *}$, DEEPALA V S G K SHARMA ${ }^{\mathrm{a}}$, PINNELLI S R PRASAD ${ }^{\mathrm{a}}$ \\ and S RAMANA MURTHY ${ }^{\mathrm{b}}$ \\ ${ }^{a}$ Gas Hydrates group, National Geophysical Research Institute (Council for Scientific \& Industrial Research), \\ Hyderabad 500 007, India \\ ${ }^{\mathrm{b}}$ Department of Physics, Osmania University, Hyderabad 500 007, India
}

MS received 25 October 2011; revised 5 June 2013

\begin{abstract}
This work presents the measurement of thermal conductivity of nano-silica particles using needle probe method. The validation test of thermal probe was conducted on ice and THF hydrates using our experimental set up and the results are satisfactory when compared with the literature data. The nano silica used in this study is with particle sizes in the range 50-1000 $\mathrm{nm}$. The sand powders sieved in different sizes $<75$ and $75 \mu \mathrm{m}>d>250 \mu \mathrm{m}$ were also studied to probe the particle size dependence on thermal conductivity. Thermal conductivity decreased by about $70 \%$ in silica nano powders.
\end{abstract}

Keywords. Thermal conductivity; micro and nano particle size; needle probe method.

\section{Introduction}

The thermal conductivity in sand depends upon various factors, i.e. on particle size distribution, structure, porosity, saturation degree and temperature. Amongst all these factors, porosity and saturation degree have remarkable effects on thermal conductivity (Chen 2008). Our study focuses mainly on the effects of particle size on thermal conductivity of silica. Several methods have been used to measure thermal conductivity of soils and details of these methods have been presented (Donazzi 1977). The methods can be divided into two major categories: steady heat-flow method and transient heat-flow method. The steady heat-flow method generally requests a long testing time, consequently, steady heat-flow method is not suitable in some cases. On the contrary, the transient heat-flow method is rapid and only a small temperature increment occurs in the process of test. The transient thermal probe method is a transient heat-flow method. It determines the thermal conductivity of medium through measuring the rate of temperature rise caused by an infinite line heat source within an infinite homogeneous medium. Thermal probe method of determining conductivity is the best method available because of its easy design and usage. The thermal probe can be used to test specimens in the laboratory or soils in situ.

\section{Thermal probe construction and method}

The thermal probe used in this work was constructed from seamless thin-walled stainless steel tubes, $100 \mathrm{~mm}$ long and $3 \mathrm{~mm}$ outer diameter. The main probe comprises of a nicrome

\footnotetext{
*Author for correspondence (dhanu_jay2000@yahoo.com)
}

heater wire and a thermocouple as shown in figure 1. The probe assembly design is in accordance with ASTM D 5334 (Cars law and Jaeger 1964). Insulated nicrome heater wire, $0.5 \mathrm{~mm}$ in diameter, was looped and inserted into the full length of the tube to form the heating element; Pt100 thermocouple was inserted midway along the length of a tube. Both the ends of heating element and thermocouple wires protruded from the same end of the tube. The tube was filled with thermal binding system (TBS20S, Electrolube, UK) which is thermally active but electrically insulated and a metal tip was placed on an open end of the tube. The epoxy was allowed $24 \mathrm{~h}$ for setting and the extended heater wire ends were cut close to the thermal probe and power supply leads were soldered to them. The probe normally consisted of an electrical heater, a temperature sensor and a thermocouple. The probe was inserted into an isothermal material and powered. The time-temperature data from the probe were analysed to obtain the mean apparent thermal conductivity, $\lambda$ for the material over the temperature range introduced in the experiment. Apparent thermal conductivities are obtained from the data using (3):

$$
\lambda=(Q / 4 \pi) /(d T / d \ln t),
$$

where $Q$ is heat generated, $d T$ the change in temperature and $d \ln t$ the natural log of time. Equation (1) indicates an attractive method for thermal conductivity measurements with the advantages of low cost and short test time.

The general solution for the temperature variation with time of a cylinder of a perfect conductor with small radius transferring heat at a constant rate to the surrounding isothermal infinite medium with constant properties indicates that in all the cases, a plot of temperature against $\ln t$ has a linear asymptote of slope $(Q / 4 \pi A)$ (Cars law and Jaeger 1964). 
Therefore, if the value of $Q$ is known, apparent thermal conductivity $\lambda$ is determined. A constant current, $I$, is supplied to a heater wire with resistance $R$, per meter. The heater wire's output per meter, $Q$, is determined from $Q=2 I^{2} R$, where factor 2 accounts for the heater wire being a loop running the length of the needle probe. From Blackwell (1954), the heater wire's output causes the temperature change measured within the probe, $\Delta T$, to vary as:

$$
\Delta T=A \ln (t)+B,
$$

where $t$ is the time in seconds since the probe began heating the sample, $\lambda$ the thermal conductivity and $A$ the slope.

$$
\lambda=1 / A \cdot Q / 4 \pi .
$$

Equation (3) indicates an attractive method for thermal conductivity measurement with the advantages of low cost and short test time. Hence, this method would be ideal for conducting many tests per day (Manohar et al 2000). In practical situations, the finite radius of the probe has the effect of time delay before the theoretical rate of radial heat flow through the surface of the probe is equal to the heat dissipated by the

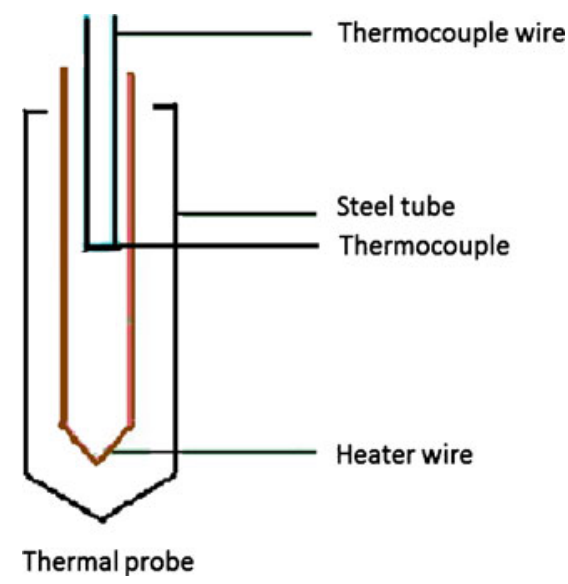

Figure 1. Needle probe arrangement.

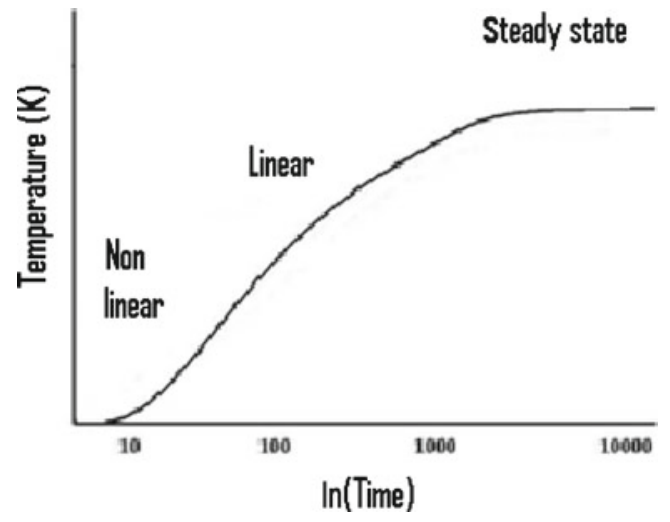

Figure 2. Graph between time and temperature. heater filament (Winterport 1970). Therefore, for practical situations, the general shape of $T-\ln t$ plot includes an initial nonlinear region followed by a linear region. With the passage of time, the probe temperature will level off to a steady state value because of an isothermal specimen boundary. The general shape of a typical $T-\ln t$ plot is shown in figure 2 (Annual Book of ASTM Standards 1995).

\section{Experimental}

We assembled an experimental set up based on line heat source and an automated data logging system to measure thermal conductivity. Thermal probe method of determining conductivity is the best method available because of its easy design and usage. The thermal probe was powered by a regulated d.c. power supply. The electrical circuit used is shown schematically in figure 3. The system employed Aplab 7642 precision power supply voltage from 0 to $20 \mathrm{~V}$. Temperaturetime variation of the thermocouple in the thermal probe was monitored and recorded by the computer through $I / O$ board. The computer was programmed to sense one temperature signal per $3 \mathrm{~s}$, computes the average and display it for each $3 \mathrm{~s}$ and stores display readings of time and temperature for a total of 200-400 entries. Reliability tests were conducted on ice and THF hydrates to validate the experimental set up and the results are corroborated with the literature. The silica nano powder used was obtained from Intelligent Materials Pvt. Ltd. (NANOSHEL) and used as such. SEM pictures of the silica samples are shown in figure 4. In our experiments, we used $50 \mathrm{~g}$ of silica in each particle size. Silica filled into the cell with as minimum as possible air gaps. The probe was inserted exactly into the middle of the cell (sample) and the probe is energized with d.c. voltage. The analysis characterized by (1) required that the specimen be isothermal, therefore, during the experimental study, the total experimental set up was covered with thermally insulated box, thus limiting in situ usage.

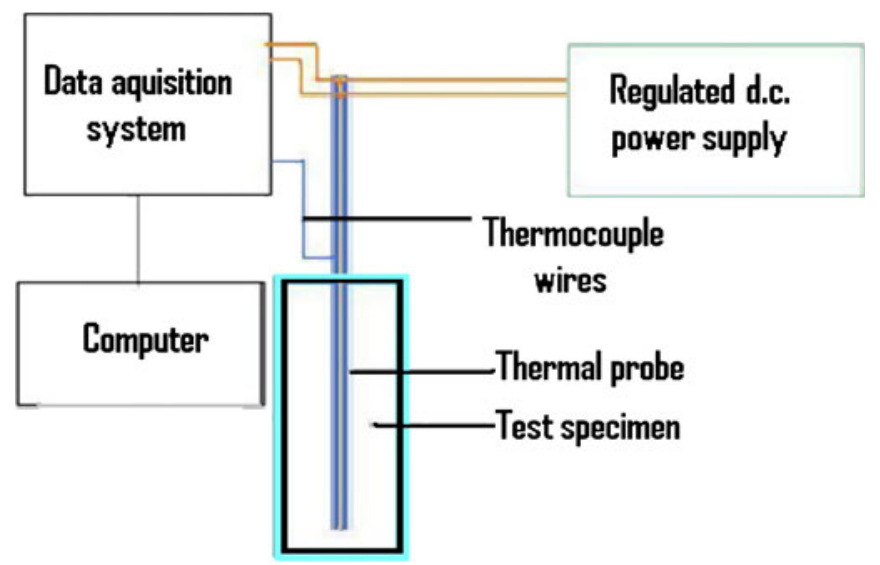

Figure 3. Schematic diagram of electrical circuit and data acquisition system. 

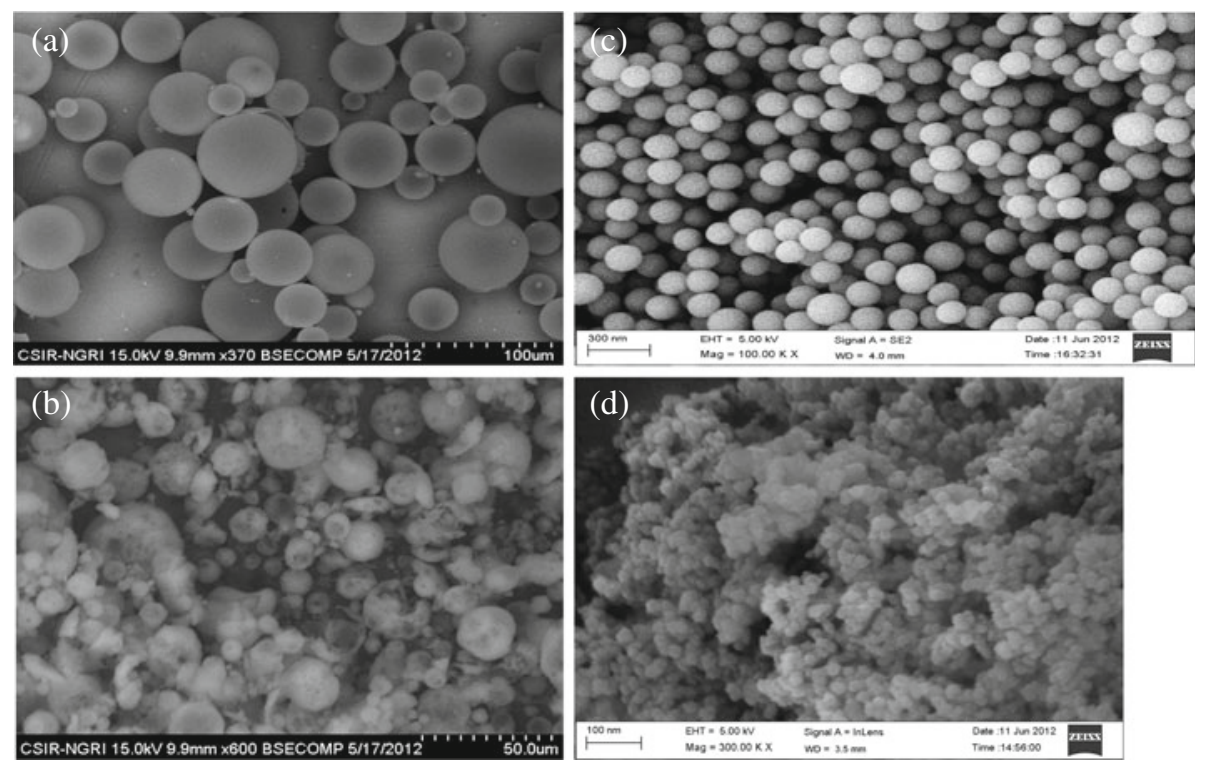

Figure 4. (a) $<75 \mu \mathrm{m}$, (b) $1000 \mathrm{~nm}$, (c) $250 \mathrm{~nm}$ and (d) $50 \mathrm{~nm}$ are SEM images of samples used.

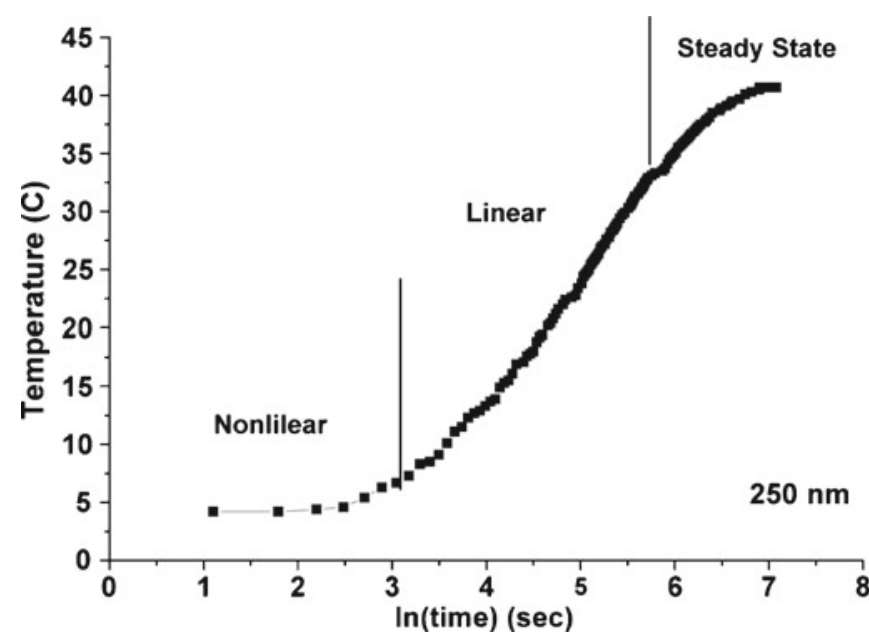

Figure 5. Typical thermal conductivity curve of $250 \mathrm{~nm}$ silica.

The experimental procedure involved, inserting the probe in the material to be tested after both were in thermal equilibrium with the surroundings. Since the friction associated with inserting the probe may cause a measurable temperature increase, the probe temperature was monitored to make certain that isothermal conditions were attained before the heater was powered. This thermal equilibration usually takes 5-10 min. The data acquisition system and heater power were turned on together once the thermal equilibrium had been achieved. The data acquisition system was used to record probe current, temperature and time for $\sim 600$ 1200 s. Since nicrome wire has a negligible change in electrical resistance with temperature (Weast 1982), the power to the probe heater remains constant. A linear fit to a linear expression for $T$ in terms of ' $\ln t$ ' was used to describe linear

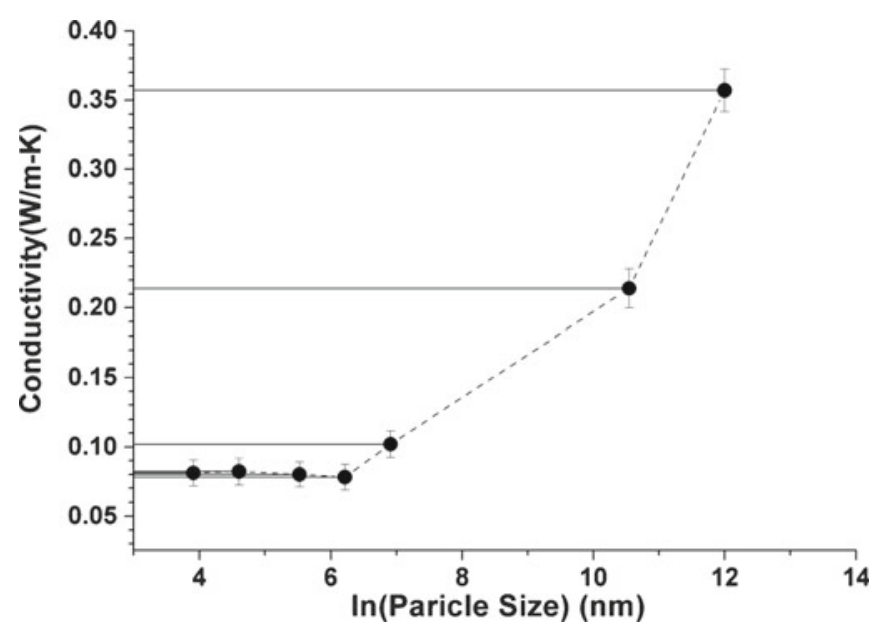

Figure 6. Graph between log of particle size and thermal conductivity.

segment of the heat-up curve which is used while calculating the slope of the curve.

\section{Results and discussion}

Thermal conductivity and viscosity affect heat transfer behaviour of nanofluids in opposite ways. As a result, combination of thermal conductivity enhancement and increment of the viscosity can give either enhancement or deterioration of the heat transfer coefficient (Ding et al 2007). The thermal conductivity of the nano-scale silicon particles increases with their diameter and is two orders of magnitude lower than that of bulk silicon, this may be due to particle phase boundary (Fang et al 2006). From the studies of 


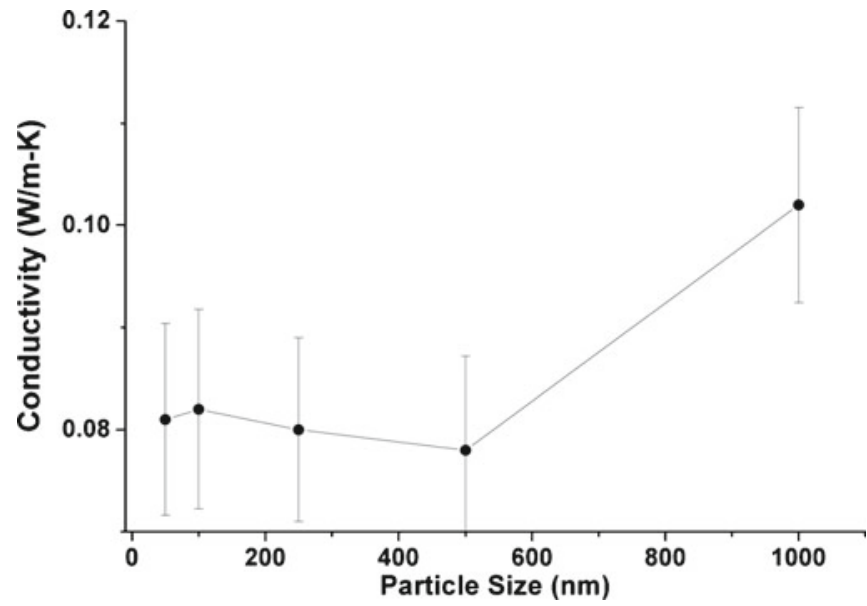

Figure 7. Thermal conductivity at nano level.

Table 1. The dependency of thermal conductivity with different silica particle sizes.

\begin{tabular}{rcc}
\hline $\begin{array}{l}\text { Particle size } \\
(\mathrm{nm})\end{array}$ & $\begin{array}{c}\text { Conductivity } \\
(\mathrm{W} / \mathrm{m} \cdot \mathrm{K})\end{array}$ & $\begin{array}{c}\text { std error } \\
( \pm)\end{array}$ \\
\hline 162500 & $0 \cdot 357$ & 0.0592 \\
38000 & $0 \cdot 214$ & 0.0301 \\
1000 & $0 \cdot 102$ & 0.00855 \\
500 & $0 \cdot 078$ & 0.00821 \\
250 & $0 \cdot 080$ & 0.0081 \\
100 & 0.082 & 0.00877 \\
50 & $0 \cdot 081$ & 0.00823 \\
\hline
\end{tabular}

Al Nakshabandi and Kohnke (1965), it was observed that the variation in the thermal conductivity was negligible when the particle size exceeded $1000 \mu \mathrm{m}$, whereas in case of nano size a significant variation in thermal conductivity was observed. We measured thermal conductivity in dry samples of nano silica to observe how the conductivity varies from micro to nano. It was observed that there is a decrease in thermal conductivity with decrease in particle size of nano-sized particles as compared to micro sized particles and this increase in thermal conductivity of micro sized particles can be attributed to their high surface to volume ratio. Moreover, the decrease in thermal conductivity with decrease in particle size is probably due to the created void spaces in the sample which is completely dependent on the particle size.

The smaller grains will pack closer, forming a more homogenous specimen, so we expect higher conductivity but because of air trapped in void spaces of the system which is a bad conductor, therefore, the conductivity is reduced. The typical conductivity curve of silica, $250 \mathrm{~nm}$, is shown in figure 5 , which is divided into three parts. Nonlinear (time is too short to satisfy (1)), linear data used for linear fit which is useful for calculation of thermal conductivity and steady state (dominated by edge and end effects). Test results on silica and sand size in the range $50-1000 \mathrm{~nm}$ and different sizes $<75 \mu \mathrm{m}$ and $75 \mu \mathrm{m}>d>250 \mu \mathrm{m}$ showed a decrease in thermal conductivity. In the graph between log of silica size vs thermal conductivity (figure 6), we observed that when silica size is decreased, thermal conductivity also decreased by about $70 \%$ from micro to nano and observed to be saturated at around $500 \mathrm{~nm}$ nano level with small variations up to $50 \mathrm{~nm}$ (shown in figure 7). Table 1 shows values of particle size and the corresponding thermal conductivity with associated standard error.

\section{Conclusions}

We studied thermal conductivity measurements using thermal probe method. Test results on silica sand size in the range $50-1000 \mathrm{~nm}$ and different sizes $<75$ and $75 \mu \mathrm{m}>$ $d>250 \mu \mathrm{m}$ showed a decrease in thermal conductivity. We observed that the thermal conductivity decreases by about $70 \%$ upon reducing silica particles size from micro to nano.

\section{References}

Al Nakshabandi G and Kohnke H 1965 Agric. Meteor. 2271

ASTM D 5334 Standard Test Method for Determination of Thermal Conductivity of Soil and Soft Rock by Thermal Needle Probe Procedure, 1995 Annual Book of ASTM Standards, Vol. 04.09, p. 225

Blackwell J H 1954 J. Appl. Phys. 25137

Cars law H S and Jaeger J C 1964 Conduction of heat in solids (UK: Oxford Press) 2nd ed., pp. 58-60, 344-345

Chen Shan Xiong 2008 Heat Mass Transfer 441241

Ding Yulong et al 2007 Heat transfer intensification using nanofluid, KONA No. 25

Donazzi F 1977 Proc. IEE 123506

Fang Kuan-Chuan, Weng Cheng-I and Ju Shin-Pon 2006 Nanotechnology 173909

Manohar K, Yarbrough David W I and Booth James R 2000 Test. Eval. 28(5) 345

Weast R C ed., 1982 CRC hand book of chemistry and physics (CRC Press Inc.) 62nd ed., p. E-82

Winterport H F 1970 Suggested method of test for thermal resistivity of soil by thermal probe, Special procedure for testing soil and rock for engineering purposes, ASTM STP 479, ASTM p. 264 